\title{
Critical role of protein L-isoaspartyl methyltransferase in basic fibroblast growth factor-mediated neuronal cell differentiation
}

\author{
To Thi Mai Dung ${ }^{1, \#}$, Young-Su Yi, $i^{1,2, \#}$, Jieun Heo ${ }^{1}$, Woo Seok Yang ${ }^{1}$, Ji Hye Kim ${ }^{1}$, Han Gyung Kim ${ }^{1}$, Jae Gwang Park ${ }^{1}$, \\ Byong Chul Yoo ${ }^{3}$, Jae Youl Cho ${ }^{1, *}$ \& Sungyoul Hong ${ }^{1, *}$ \\ ${ }^{1}$ Department of Genetic Engineering, Sungkyunkwan University, Suwon $16419,{ }^{2}$ Department of Pharmaceutical Engineering, Cheongju \\ University, Cheongju 28503, ${ }^{3}$ Colorectal Cancer Branch, Research Institute, National Cancer Center, Goyang 10408, Korea
}

\begin{abstract}
We aimed to study the role of protein L-isoaspartyl methyltransferase (PIMT) in neuronal differentiation using basic fibroblast growth factor (bFGF)-induced neuronal differentiation, characterized by cell-body shrinkage, long neurite outgrowth, and expression of neuronal differentiation markers light and medium neurofilaments (NF). The bFGF-mediated neuronal differentiation of PC12 cells was induced through activation of mitogen-activated protein kinase (MAPK) signaling molecules [MAPK kinase 1/2 (MEK1/2), extracellular signal-regulated kinase 1/2 (ERK1/2), and p90RSK], and phosphatidylinositide 3-kinase (PI3K)/Akt signaling molecules PI3Kp110 $\beta$, PI3Kp110 $\gamma$, Akt, and mTOR. Inhibitors (adenosine dialdehyde and S-adenosylhomocysteine) of protein methylation suppressed bFGF-mediated neuronal differentiation of PC12 cells. PIMTeficiency caused by PIMT-specific siRNA inhibited neuronal differentiation of PC12 cells by suppressing phosphorylation of MEK1/2 and ERK1/2 in the MAPK signaling pathway and Akt and $\mathrm{mTOR}$ in the PI3K/Akt signaling pathway. Therefore, these results suggested that PIMT was critical for bFGF-mediated neuronal differentiation of PC12 cells and regulated the MAPK and Akt signaling pathways. [BMB Reports 2016; 49(8): 437-442]
\end{abstract}

\section{INTRODUCTION}

Neuronal differentiation is a complex process in which nerve cells acquire specific biochemical, physiological, and

*Corresponding authors. Jae Youl Cho, Tel: +82-31-290-7868; Fax: +82-31-290-7870; E-mail: jaecho@skku.edu, Sungyoul Hong, Tel: +82-31-290-7862; Fax: +82-31-290-7870; E-mail: syhong@skku.edu ${ }^{\text {"}}$ These authors contributed equally to this work.

http://dx.doi.org/10.5483/BMBRep.2016.49.8.020

Received 1 February 2016, Revised 16 February 2016, Accepted 10 March 2016

Keywords: Akt, Basic fibroblast growth factor, Mitogen-activated protein kinase, Neuronal differentiation, Phosphatidylinositide 3-kinase, Protein L-isoaspartyl methyltransferase morphological properties during development. Differentiation involves many molecules including growth factors, intracellular signaling molecules, and transcription factors (1). Basic fibroblast growth factor (bFGF) is a potent neurogenic factor that regulates neuronal differentiation and development through activation of extracellular signal-regulated kinase (ERK) and early transcription factors, activator protein (AP)-1, and nuclear factor $(\mathrm{NF})-\kappa \mathrm{B}(2,3)$. However, the detailed molecular mechanisms of bFGF-mediated neuronal differentiation and development, which vary among cell types, are still unclear.

bFGF is a member of the fibroblast growth factor family, which functions in a variety of biological processes including cell growth, differentiation, and survival. bFGF was first used for research on the neuronal differentiation of bone marrow stromal cells (4). A number of in vitro and in vivo studies revealed bFGF functions in neuronal differentiation (5-8). bFGF reportedly induces neuronal differentiation of cells of neuronal origin and non-neuronal origin such as bone marrow stromal cells (4), adrenal chromaffin cells (7), retina epithelial cells (9), and retinal pigment cells (10). These results suggest that bFGF has a potent neuronal-inductive function. bFGF reportedly activates several downstream signaling pathways through binding with FGF receptors (FGFRs) $(11,12)$. Activated pathways include the signaling cascades of phospholipase C- $\gamma$, Src, Crk, SNT1/FRS2 (13), MAPK/ERK $(8,10,14)$, mTOR (15), and RhoA (16). Although bFGF reportedly modulates these signaling pathways, the pathways critical for bFGF-mediated neuronal differentiation are unknown. In addition, whether bFGF activates unknown signaling pathways in bFGF-mediated neuronal differentiation still needs to be elucidated.

Protein L-isoaspartyl methyltransferase (PIMT), a member of the protein methyltransferase family, is a protein-repair enzyme that methylates isoaspartyl residues generated by asparagine deamidation. From aged proteins, it was found that PIMT plays a critical role in recovering function of aged protein (17-19). Because of the relevance of PIMT function for asparagine deamidation of proteins, most previous studies on PIMT have investigated its functions in aged protein repair $(17,20)$. A number of studies, however, report that asparagine deamidation is involved a variety of biological functions in addition to protein repair, including interaction with the 
extracellular matrix $(21,22)$, apoptosis (23-26), synaptic transmission (27), and lifespan extension (28, 29). Recent studies report that protein arginine methyltransferase (PRMT), another member of the protein methyltransferase family, is involved in regulating neuronal differentiation and development (30-32). These findings strongly indicate that protein methyltransferase is critical for the regulation of neuronal differentiation and development, and PIMT, which is in the same protein methyltransferase family, is also involved in neuronal differentiation and development.

This study investigated how bFGF induces neuronal differentiation in PC12 cells and the cellular molecules critical for this process. We showed that both MAPK and PI3K/Akt signaling pathways are specifically activated in bFGF-mediated neuronal differentiation of PC12 cells. We demonstrated that PIMT, a member of the protein methyltransferase family, is crucial for bFGF-mediated neuronal differentiation of PC12 cells and regulates the activity of signaling molecules in the MAPK and PI3K/Akt pathways.

\section{RESULTS AND DISCUSSION}

We confirmed neuronal differentiation by bFGF using PC12 cells. PC12 cells were treated with bFGF and morphological changes were monitored every 2 days for 6 days. In accordance with previous studies, bFGF induced neuronal differentiation of PC12 cells. Morphological changes of bFGF-treated PC12 cells included shrinkage of cell bodies and formation of long neurite outgrowths connecting to neighboring cells (Fig. 1A). These morphology changes started on day 2 with full changes after day 4 and up to day 6 after bFGF treatment (Fig. 1A). NFs in neuronal cells are differentiation

A

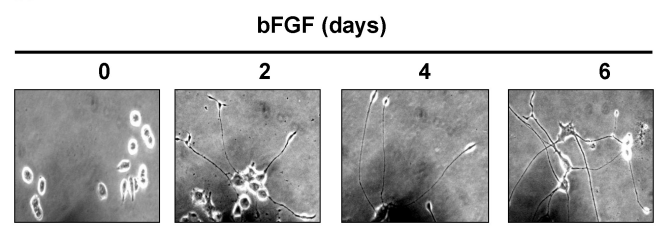

B

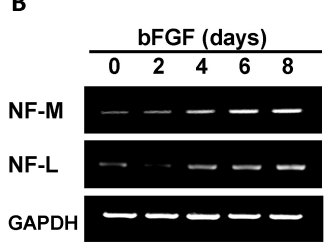

C

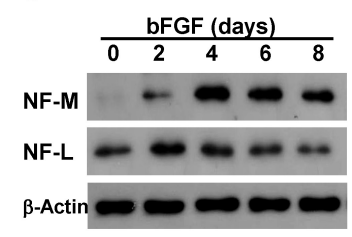

Fig. 1. bFGF induces neuronal differentiation of PC12 cells. (A) Neuronal differentiation of PC12 cells was induced by treatment with $50 \mathrm{ng} / \mathrm{ml}$ bFGF. (B, C) The mRNA and protein levels of NF-M and NF-L from PC12 cells treated with $50 \mathrm{ng} / \mathrm{ml}$ bFGF for indicated times were analyzed by RT-PCR and Western blot. markers and a major component of the neuronal cytoskeleton. NFs are believed to provide structural support for axons and modulate axon diameters (33). To examine whether NF expression was induced in cells differentiated by bFGF, expression of NF-M and NF-L in bFGF-treated PC12 cells was determined. NF-M and NF-L mRNA gradually increased from day 2 to day 8 in bFGF-treated PC12 cells (Fig. 1B). NF-M and NF-L protein were also induced in PC12 cells with bFGF treatment; however, protein expression patterns were different from mRNA patterns. NF-M protein gradually increased up to day 4 with bFGF treatment and decreased afterward (Fig. 1C). NF-L protein was immediately induced, peaked at day 2 after bFGF treatment and gradually decreased afterward (Fig. 1C). The reason for the different mRNA and protein expression patterns was unclear, but might be explained by protein stability. These results indicated that bFGF induced neuronal differentiation of PC12 cells.

To investigate the molecular mechanisms by which bFGF induced neuronal differentiation, the activities of intracellular signaling molecules were examined in bFGF-treated PC12 cells. bFGF binding to FGFR modulates cellular functions including cell proliferation, survival, and motility through activation of intracellular signaling pathways such as MAPK and PI3K (34). Therefore, MAPK and PI3K/Akt signaling pathways were examined in bFGF-treated PC12 cells during neuronal differentiation. The MAPK pathway intracellular signaling molecules, MEK1/2, ERK1/2, and p90RSK, were activated in PC12 cells treated with bFGF (Fig. 2A). PI3K/Akt pathway signaling molecules were also triggered by bFGF. PI3Kp110 $\beta$ and PI3Kp110 $\gamma$ were gradually activated with bFGF treatment while Akt was rapidly activated before decreasing (Fig. 2B). Although both PI3Ks and Akt were activated by bFGF in PC12 cells, the molecules had different activation patterns, suggesting that PI3Ks and Akt are activated by bFGF in distinct ways. To determine if MAPK and PI3K/Akt signaling pathways are critical for bFGF-mediated neuronal differentiation of PC12 cells, the modulation of cell-body shrinkage and neurite outgrowths was examined in the presence of the MAPK inhibitor U0126 or the PI3K/Akt inhibitor LY294002. PC12 cells exhibited morphological changes with bFGF alone and these changes were suppressed in bFGF-treated PC12 cells in the presence of U0126 or LY294002 (Fig. 2C). NF-M expression induced by bFGF was also decreased by U0126 or LY294002 (Fig. 2D). The activity of mTOR, a downstream molecule regulated by PI3K/Akt signaling pathways (35), was also examined in bFGF-treated PC12 cells. Phosphorylation of mTOR was induced by bFGF in PC12 cells (Fig. 2E). Morphological changes induced by bFGF were significantly suppressed when mTOR was inhibited by the specific inhibitor rapamycin (Fig. 2F upper). NF-M protein upregulation by bFGF was also dose-dependently reduced by rapamycin (Fig. 2E lower). Cross-specificity tests of the inhibitors confirmed that each was specific for its target and did not affect other signaling pathways (data not shown). These results suggested that 
A

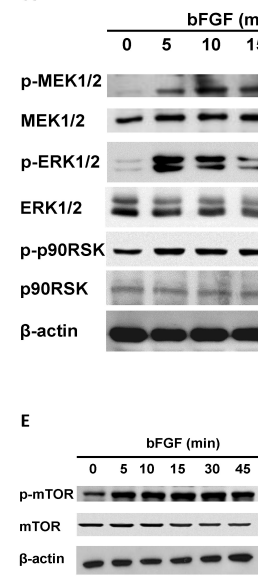

B

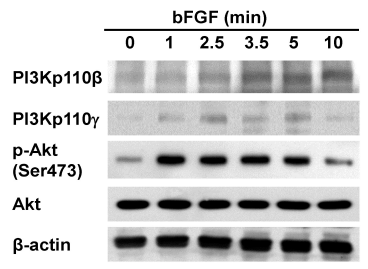

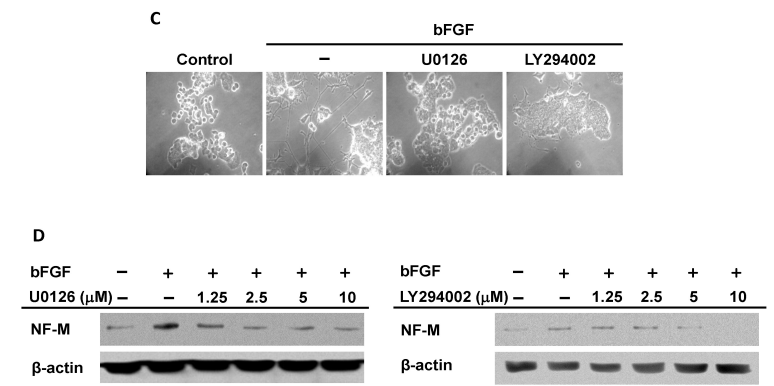

$\mathrm{F}$
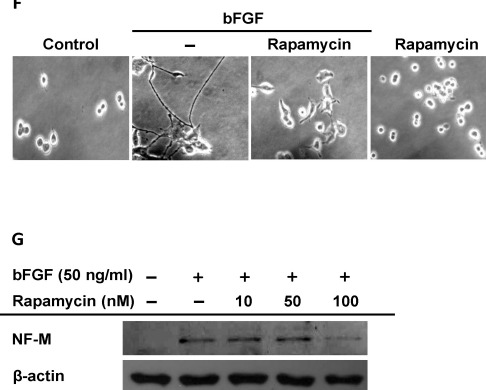

B-actin

bFGF-mediated neuronal differentiation of PC12 cells was specifically regulated by the signaling pathways. These results indicated that bFGF induced neuronal differentiation of PC12 cells through activation of both the MAPK and PI3KAkt signaling pathways.

Protein methylation is a post-translational modification known to regulate signal transduction pathways similar to phosphorylation. Cimato et al., reported that nerve growth factor modulates methylation of several proteins and protein methylation is involved in neuronal differentiation (36). Therefore, we explored protein methylation in bFGF-mediated neuronal differentiation. For this, PC12 cells were treated with bFGF in the absence or presence of protein-methylation inhibitors adenosine dialdehyde (AdOx) and S-adenosyl L-homocysteine $(\mathrm{SAH})$, and neuronal differentiation pattern of PC12 cells was examined. Morphological changes of cell-body shrinkage and extended neurite outgrowth induced by bFGF were suppressed by the inhibitors (Fig. 3A). Expression of neuronal differentiation markers NF-L and neuron-specific class III beta-tubulin (Tuj-1) was examined in PC12 cells treated with inhibitors. Expression of NF-L and Tuj-1 was induced by bFGF and dose-dependently decreased with protein methylation inhibitors (Fig. 3B). Previous studies reported that protein methyltransferases, such as histone methyltransferase and PRMTs are involved in neuronal differentiation and development through regulation of protein methylation $(37,38)$. Based on these observations, the protein methyltransferase PIMT was examined. PIMT-deficient PC12 cells were generated by transfecting PC12 cells with three different PIMT-specific siRNAs; expression was measured by Western blot. All

Fig. 2. bFGF-mediated neuronal differentiation of PC12 cells activates MAPK and PI3K/Akt signaling pathways. (A, B, D, E, G) Levels of phosphorylated and total MEK1/2, ERK1/2, p90RSK, PI3Kp110 $\beta$, PI3Kp110 $\gamma$, Akt, NF-M, and mTOR from whole lysates of PC12 cell treated with $50 \mathrm{ng} / \mathrm{ml}$ bFGF in the absence or presence of U0126 (D left), LY294002 (D right) or rapamycin (G) for 3 days were analyzed by Western blotting. (C, F) Neuronal differentiation of PC12 cells was induced by treatment with $50 \mathrm{ng} / \mathrm{ml} \mathrm{bFGF} \mathrm{in} \mathrm{the} \mathrm{absence} \mathrm{or} \mathrm{presence} \mathrm{of} \mathrm{U0126} \mathrm{(10}$ $\mu \mathrm{M})$, LY294002 $(50 \mu \mathrm{M})$ or rapamycin (100 nM) for 6 days.

PIMT-specific siRNAs inhibited PIMT expression. PIMT siRNA 3 was most effective at inhibiting PIMT (Fig. 3C) and was used for subsequent experiments. PIMT-deficient PC12 cells were treated with bFGF and morphological changes were examined. Morphological changes were induced by bFGF in SCRransfected control PC12 cells and changes were significantly suppressed by bFGF in PIMT-deficient PC12 cells (Fig. 3D). Expression of Tuj-1 was also determined in PIMT-deficient PC12 cells. In accordance with the morphology changes, Tuj-1 expression increased with bFGF in control PC12 cells and expression was significantly decreased in PIMT-deficient cells (Fig. 3E). These results suggested that PIMT is critical for bFGF-mediated neuronal differentiation of PC12 cells through regulation of protein methylation.

Since bFGF induced neuronal differentiation of PC12 cells through activation of MAPK and PI3K/Akt signaling pathways (Fig. 2), we investigated how PIMT regulated these pathways in bFGF-mediated neuronal differentiation of PC12 cells. The activities of MAPK and PI3K/Akt pathway signaling molecules were determined in PIMT-deficient PC12 cells. Phosphorylation of in MAPK pathway signaling molecules c-Raf, MEK1/2, ERK1/2, and p90RSK was determined by Western blot. Phosphorylation of MEK1/2 and ERK1/2 was suppressed while phosphorylation of c-Raf was unchanged in PIMT-deficient PC12 cells treated with bFGF (Fig. 4A). The activities of $\mathrm{PI} 3 \mathrm{~K} / \mathrm{Akt}$ pathway signaling molecules were also measured in PIMT-deficient PC12 cells. Akt phosphorylation was significantly decreased while PI3Kp110 $\beta$, PI3Kp110 PDK1 were not changed in PIMT-deficient PC12 cells treated with bFGF (Fig. 4B). Akt, PI3Kp110ß, and PI3Kp110y were 


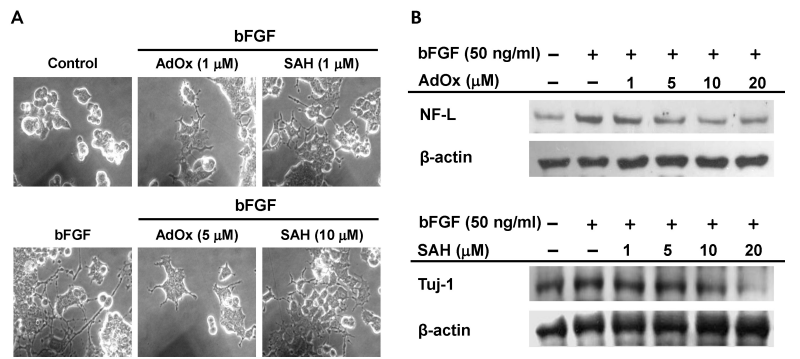

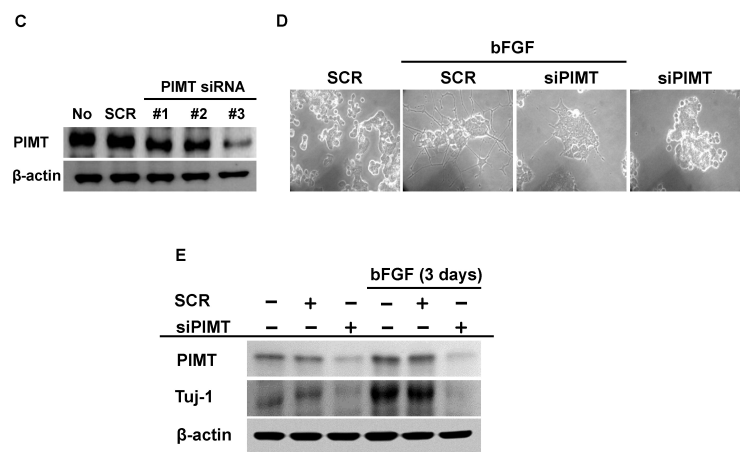

Fig. 3. PIMT induces bFGF-mediated neuronal differentiation of PC12 cells. (A, D) Neuronal differentiation of PC12 cells was triggered by bFGF $(50 \mathrm{ng} / \mathrm{ml})$ treatment in the absence or presence of AdOx (1 and $5 \mu \mathrm{M})$, SAH (1 and $10 \mu \mathrm{M})$ or under transfection with control SCR or PIMT siRNA for 6 days. (B, C, E) NF-M, Tuj-1, and PIMT from whole lysates of PC12 cells treated with bFGF in the absence or presence of $\mathrm{AdOx}$ (upper) and SAH (lower) for 3 days or under transfection with 20 nM control SCR siRNA or PIMT siRNA (1-3) for 2 (C) or 3 (E) days were detected by Western blot.

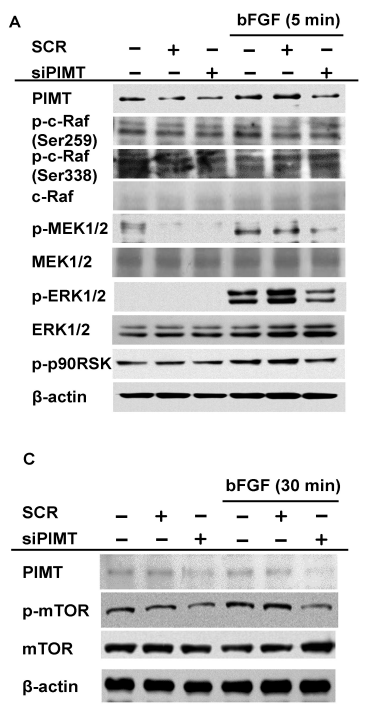

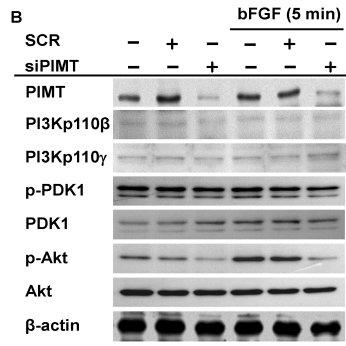

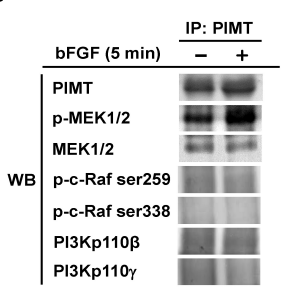

$E$

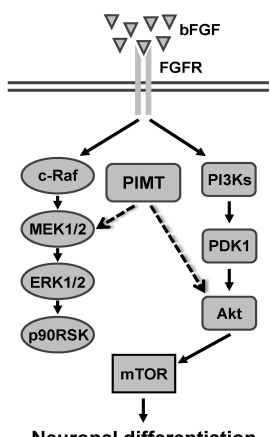

Neuronal differentiation
Fig. 4. PIMT induces bFGF-mediated neuronal differentiation of $\mathrm{PC} 12$ cells by activating MAPK and Akt signaling pathways. (A-C) Phosphorylated and total forms of c-Raf, MEK1/2, ERK1/2, p90RSK, PI3Kp110b, PI3Kp110g, PDK1, Akt, and mTOR from PC12 cells, transfected with control SCR or PIMT siRNA for $48 \mathrm{~h}$, treated with $50 \mathrm{ng} / \mathrm{ml}$ bFGF for 5 or 30 min were detected by Western blot. (D) Binding level of MEK1 with PIMT was evaluated by immunoprecipitation with anti-PIMT from whole lysates of PC12 cells treated with vehicle or $50 \mathrm{ng} / \mathrm{ml}$ bFGF for 5 min. (E) Proposed model for the role of PIMT in bFGF-mediated neuronal differentiation of PC12 cells. activated in PC12 cells by bFGF (Fig. 2B), indicating that PIMT specifically targeted and regulated the activity of only Akt, which is downstream of PI3Ks in the PI3K/Akt signaling pathway. In addition to PIMT, another mechanism might regulate the PI3K/Akt signaling pathway by activating PI3Kp110 3 and PI3Kp110 in bFGF-mediated neuronal differentiation. Phosphorylation of mTOR, which is downstream in the Akt signaling pathway, was inhibited in PIMT-deficient PC12 cells treated with bFGF (Fig. 4C). Since PIMT clearly regulated the activities of MAPK signaling pathways in bFGF-treated PC12 cells, we examined whether PIMT interacted with one of the upstream MAPK signaling molecules, MEK $1 / 2$, by immunoprecipitation and Western blotting. As we expected, PIMT interacted with phosphorylated and total forms of MEK $1 / 2$ but not with phosphorylated c-Raf, PI3Kp110 $\beta$, and PI3Kp110 $\gamma$ in the MAPK and Akt pathways (Fig. 4D). These results indicated that MEK $1 / 2$ and Akt might be PIMT substrates for transducing bFGF-mediated signaling to downstream pathways. These results suggested that PIMT induced bFGF-mediated neuronal differentiation of PC12 cells by modulating the activities of MEK $1 / 2$ and ERK $1 / 2$ in the MAPK and Akt signaling pathways.

In summary, this study showed that bFGF induced neuronal differentiation of PC12 cells through the activation of the MAPK and PI3K/Akt signaling pathways. PIMT was crucial for bFGF-mediated neuronal differentiation of PC12 cells through 
regulating MEK1/2 and ERK1/2 activities in the MAPK and Akt signaling pathways (Fig. 4E). This study proposes a new role for the protein methyltransferase PIMT in neuronal differentiation and provides new information for understanding the molecular mechanism of neuronal differentiation regulation and factors critical for this event. PIMT could be a target for diseases with neuronal differentiation defects.

\section{MATERIALS AND METHODS}

\section{Materials}

PC12 cells were from the Korea Cell Line Bank (Seoul, Korea). Roswell Park Memorial Institute 1640 (RPMl1640) cell culture medium, fetal bovine serum (FBS), phosphate-buffered saline (PBS), streptomycin, penicillin, and L-glutamine were from Gibco (Grand Island, NY, USA). Phosphorylated-protein and total protein-specific antibodies [neurofilament-medium (NF-M), NF-light (L), MEK1/2, ERK1/2, p90RSK, PI3Kp110ß, PI3Kp110\%, Akt, mTOR, Tuj-1, PIMT, c-Raf, PDK1, and $\beta$-actin] were from Cell Signaling Technology (Beverly, MA, USA). bFGF, AdOx, and SAH were from Sigma-Aldrich (St. Louis, MO, USA). U0126, LY294002, and rapamycin were from Calbiochem (La Jolla, CA, USA). Lipofectamine 2000 reagent and siRNAs specific for PIMT were from Thermo Fisher Scientific (Waltham, MA, USA). Protein A-Sepharose beads were from GE Healthcare Life Sciences (Pittsburgh, PA, USA). PCR primers were synthesized by and PCR premix was from Bioneer Inc. (Daejeon, Korea).

Cell culture and in vitro neuronal differentiation of PC12 cells PC12 cells were cultured in RPMI 1640 medium containing $10 \%$ heat-inactivated FBS, $100 \mu \mathrm{g} / \mathrm{ml}$ streptomycin, $100 \mathrm{U} / \mathrm{ml}$ penicillin, and $2 \mathrm{mM}$ L-glutamine at $37^{\circ} \mathrm{C}$ in a $5 \% \mathrm{CO}_{2}$ humidified incubator. Cells were split once a week and medium refreshed three times per week. For neuronal differentiation, PC 12 cells were collected by centrifugation $(1000 \times \mathrm{g}$ for $3 \mathrm{~min}$ at RT) and washed once with $1 \mathrm{X}$ PBS. Cells were resuspended in RPMI 1640 culture medium with and incubated with bFGF as indicated. Neuronal differentiation was determined by observing morphological changes such as shrinkage of cell bodies, neurite outgrowth, and NF expression.

\section{PIMT siRNA transfection}

PC12 cells were plated in antibiotic-free RPMI 1640 medium $24 \mathrm{~h}$ before transfection and transiently transfected with either control scrambled siRNA (SCR) or PIMT-specific siRNA (siPIMT) using Lipofectamine 2000 reagent according to the manufacturer's instructions. At $48 \mathrm{~h}$ after transfection, cells were to determine PIMT expression, signaling pathways, and neuronal differentiation. PIMT siRNA sequences are in Table 1.

\section{Reverse transcriptase polymerase chain reaction and PCR amplification of target genes}

PC12 cells were treated with bFGF for indicated times. After discarding media, total RNA was extracted using TRI reagent according to the manufacturer's instructions. Extracted RNA was used immediately or stored at $-80^{\circ} \mathrm{C}$. CDNA was synthesized from $1 \mu \mathrm{g}$ total RNA using reverse transcriptaseolymerase chain reaction (RT-PCR) premix (39) and used to amplify target NF-L and NF-M genes using target-specific primers. PCR primer sequences are in Table 2.

\section{Westem blots and immunoprecipitation}

Whole-cell lysates of PC12 cells were prepared by lysing cells as reported previously $(40,41)$. Protein samples were used immediately or stored at $-20^{\circ} \mathrm{C}$. For Western blots, PC12 lysates were subjected to SDS-polyacrylamide electrophoresis and transferred to polyvinylidene difluoride membranes. Phosphorylated and total target proteins were detected using specific antibodies and immune complexes were visualized using an enhanced chemiluminescence system according to the manufacturer's instruction. For immunoprecipitation, equal amounts $(500 \mu \mathrm{g})$ of whole-cell lysates from PC12 cells treated with or without $50 \mathrm{ng} / \mathrm{ml}$ bFGF for 5 min were precleared with $20 \mu$ protein A-Sepharose beads $(50 \% \mathrm{v} / \mathrm{v})$ for $1 \mathrm{~h}$ at $4{ }^{\circ} \mathrm{C}$ with gentle rotation. Lysates were then incubated with $1 \mu \mathrm{g}$ of anti-PIMT overnight at $4^{\circ} \mathrm{C}$ with gentle rotation. Immune complexes were immobilized using $20 \mu \mathrm{l}$ protein A-Sepharose beads $(50 \% \mathrm{v} / \mathrm{v})$ at $4^{\circ} \mathrm{C}$ for $4 \mathrm{~h}$ with gentle rotation and washed with pre-chilled 1X PBS five times. Immunoprecipitated proteins were analyzed by Western blot as described above using antibodies specific for phosphorylated or total MEK1/2 and Akt.

\section{REFERENCES}

1. Ma Q (2006) Transcriptional regulation of neuronal phenotype in mammals. J Physiol 575, 379-387

2. Feng $Z$ and Porter AG (1999) NF-kappaB/Rel proteins are required for neuronal differentiation of $\mathrm{SH}-\mathrm{SY} 5 \mathrm{Y}$ neuroblastoma cells. J Biol Chem 274, 30341-30344

3. Leppa S, Eriksson M, Saffrich R, Ansorge W and Bohmann D (2001) Complex functions of AP-1 transcription factors in differentiation and survival of PC12 cells. Mol Cell Biol 21, 4369-4378

4. Woodbury D, Schwarz EJ, Prockop DJ and Black IB (2000) Adult rat and human bone marrow stromal cells differentiate into neurons. J Neurosci Res 61, 364-370

5. Doniach T (1995) Basic FGF as an inducer of anteroposterior neural pattern. Cell 83, 1067-1070

6. Chiba S, Kurokawa MS, Yoshikawa H et al (2005) Noggin and basic FGF were implicated in forebrain fate and caudal fate, respectively, of the neural tube-like structures emerging in mouse ES cell culture. Exp Brain Res 163, 86-99

7. Stemple DL, Mahanthappa NK and Anderson DJ (1988) Basic FGF induces neuronal differentiation, cell division, and NGF dependence in chromaffin cells: a sequence of events in sympathetic development. Neuron 1, 517-525

8. Yang H, Xia Y, Lu SQ, Soong TW and Feng ZW (2008) Basic fibroblast growth factor-induced neuronal differentiation of mouse bone marrow stromal cells requires 
FGFR-1, MAPK/ERK, and transcription factor AP-1. J Biol Chem 283, 5287-5295

9. Pittack C, Jones M and Reh TA (1991) Basic fibroblast growth factor induces retinal pigment epithelium to generate neural retina in vitro. Development 113, 577-588

10. Galy A, Neron B, Planque N, Saule S and Eychene A (2002) Activated MAPK/ERK kinase (MEK-1) induces transdifferentiation of pigmented epithelium into neural retina. Dev Biol 248, 251-264

11. Eswarakumar VP, Lax I and Schlessinger J (2005) Cellular signaling by fibroblast growth factor receptors. Cytokine Growth Factor Rev 16, 139-149

12. Wang JK, Gao G and Goldfarb M (1994) Fibroblast growth factor receptors have different signaling and mitogenic potentials. Mol Cell Biol 14, 181-188

13. Street JT, Wang JH, Power CJ and Redmond HP (2000) Priming of neutrophil $[\mathrm{Ca} 2+] 1$ signaling and oxidative burst by human fracture fluids. J Trauma 49, 167-168

14. Abe K and Saito H (2000) Neurotrophic effect of basic fibroblast growth factor is mediated by the p42/p44 mitogen-activated protein kinase cascade in cultured rat cortical neurons. Brain Res Dev Brain Res 122, 81-85

15. Kim HJ, Kim J, Kang KS, Lee KT and Yang HO (2014) Neuroprotective Effect of Chebulagic Acid via Autophagy Induction in SH-SY5Y Cells. Biomol Ther (Seoul) 22, 275-281

16. Jeon $\mathrm{CY}$, Kim HJ, Morii $\mathrm{H}$ et al (2010) Neurite outgrowth from PC12 cells by basic fibroblast growth factor (bFGF) is mediated by RhoA inactivation through p190RhoGAP and ARAP3. J Cell Physiol 224, 786-794

17. Clarke S (2003) Aging as war between chemical and biochemical processes: protein methylation and the recognition of age-damaged proteins for repair. Ageing Res Rev 2, 263-285

18. Weintraub SJ and Deverman BE (2007) Chronoregulation by asparagine deamidation. Sci STKE 2007, re7

19. Reissner KJ and Aswad DW (2003) Deamidation and isoaspartate formation in proteins: unwanted alterations or surreptitious signals? Cell Mol Life Sci 60, 1281-1295

20. Desrosiers RR and Fanelus I (2011) Damaged proteins bearing L-isoaspartyl residues and aging: a dynamic equilibrium between generation of isomerized forms and repair by PIMT. Curr Aging Sci 4, 8-18

21. Curnis F, Cattaneo A, Longhi R et al (2010) Critical role of flanking residues in NGR-to-isoDGR transition and CD13/ integrin receptor switching. J Biol Chem 285, 9114-9123

22. Corti A and Curnis F (2011) Isoaspartate-dependent molecular switches for integrin-ligand recognition. J Cell Sci 124, 515-522

23. Deverman BE, Cook BL, Manson SR et al (2002) Bcl-xL deamidation is a critical switch in the regulation of the response to DNA damage. Cell 111, 51-62

24. Zhao R, Oxley D, Smith TS et al (2007) DNA damageinduced $\mathrm{BCl}-\mathrm{xL}$ deamidation is mediated by NHE-1 antiport regulated intracellular $\mathrm{pH}$. PLoS Biol 5, e1

25. Zhao R, Yang FT and Alexander DR (2004) An oncogenic tyrosine kinase inhibits DNA repair and DNA-damageinduced $\mathrm{Bcl}-\mathrm{xL}$ deamidation in $\mathrm{T}$ cell transformation. Cancer Cell 5, 37-49
26. Zhao R, Follows GA, Beer PA et al (2008) Inhibition of the $\mathrm{BCl}-\mathrm{xL}$ deamidation pathway in myeloproliferative disorders. N Engl J Med 359, 2778-2789

27. Bidinosti M, Ran I, Sanchez-Carbente MR et al (2010) Postnatal deamidation of $4 \mathrm{E}-\mathrm{BP} 2$ in brain enhances its association with raptor and alters kinetics of excitatory synaptic transmission. Mol Cell 37, 797-808

28. Chavous DA, Jackson FR and O'Connor CM (2001) Extension of the Drosophila lifespan by overexpression of a protein repair methyltransferase. Proc Natl Acad Sci U S A 98, 14814-14818

29. Khare S, Linster CL and Clarke SG (2011) The interplay between protein L-isoaspartyl methyltransferase activity and insulin-like signaling to extend lifespan in Caenorhabditis elegans. PLoS One 6, e20850

30. Chittka A (2013) Differential regulation of SC1/PRDM4 and PRMT5 mediated protein arginine methylation by the nerve growth factor and the epidermal growth factor in PC12 cells. Neurosci Lett 550, 87-92

31. Lin YL, Tsai YJ, Liu YF et al (2013) The critical role of protein arginine methyltransferase prmt8 in zebrafish embryonic and neural development is non-redundant with its paralogue prmt1. PLoS One 8, e55221

32. Cimato TR, Tang J, Xu Y et al (2002) Nerve growth factor-mediated increases in protein methylation occur predominantly at type I arginine methylation sites and involve protein arginine methyltransferase 1 . J Neurosci Res 67, 435-442

33. Hall GF and Yao J (2000) Neuronal morphology, axonal integrity, and axonal regeneration in situ are regulated by cytoskeletal phosphorylation in identified lamprey central neurons. Microsc Res Tech 48, 32-46

34. Goetz R and Mohammadi M (2013) Exploring mechanisms of FGF signalling through the lens of structural biology. Nat Rev Mol Cell Biol 14, 166-180

35. Nepal S and Park PH (2014) Regulatory role of autophagy in globular adiponectin-induced apoptosis in cancer cells. Biomol Ther (Seoul) 22, 384-389

36. Cimato TR, Ettinger MJ, Zhou X and Aletta JM (1997) Nerve growth factor-specific regulation of protein methylation during neuronal differentiation of PC12 cells. J Cell Biol 138, 1089-1103

37. Hong S, Heo J, Lee S et al (2008) Methyltransferase-inhibition interferes with neuronal differentiation of P19 embryonal carcinoma cells. Biochem Biophys Res Commun 377, 935-940

38. Pereira JD, Sansom SN, Smith J et al (2010) Ezh2, the histone methyltransferase of $\mathrm{PRC} 2$, regulates the balance between self-renewal and differentiation in the cerebral cortex. Proc Natl Acad Sci U S A 107, 15957-15962

39. Yoon SY, Kang HB, Ko YE et al (2015) 1-palmitoyl-2linoleoyl-3-acetyl-rac-glycerol (EC-18) Modulates Th2 Immunity through Attenuation of IL-4 Expression. Immune Netw 15, 100-109

40. Baek KS, Hong YD, Kim Y et al (2015) Anti-inflammatory activity of AP-SF, a ginsenoside-enriched fraction, from Korean ginseng. J Ginseng Res 39, 155-161

41. Kim S, Hong JW, Cho WD et al (2014) Characterization of Two Novel mÁbs Recognizing Different Epitopes on CD43. Immune Netw 14, 164-170 